\title{
¿Cuándo los maestros en formación empiezan a pensar como maestros? Un estudio inicial sobre el cambio educativo de los alumnos - maestros
}

\author{
Lylia Ana Morales Sifuentes \\ Escuela Normal Rural Ricardo Flores Magón, Saucillo, Chihuahua, México \\ lyliamorales@gmail.com \\ Blas Gómez Heredia \\ Escuela Normal Miguel Hidalgo de Parral, Chihuahua \\ blas-37@hotmail.com \\ Carlos Mario Pacheco Ríos \\ Institución Benemérita y Centenaria Escuela Normal del Estado de Chihuahua \\ carlosmario79@hotmail.com
}

\begin{abstract}
Resumen
Se presentan los resultados de un estudio exploratorio con 120 estudiantes o maestros en formación del norte de México frente a la pregunta ¿Cuál es el significado del cambio educativo para los estudiantes - profesores en las diferentes etapas de su preparación? Entre ellos se encuentran estudiantes de segundo, tercer y cuarto año de tres diferentes escuelas normales públicas quienes participaron en un programa de desarrollo profesional el cual se desarrolló en línea de preescolar y primaria utilizando un diseño de métodos mixtos, 72 estudiantes fueron seleccionados en base a su participación (alta o baja), y se entrevistó cada tres semanas durante los primeros cuatro meses del programa. Los resultados sugieren que los estudiantes - la interpretación de los maestros del significado del cambio educativo varían de su segundo año a su último año de formación pareciéndose cada vez más como la de los maestros en ejercicio, según lo reportado en la literatura que se consulta.
\end{abstract}

\section{Palabras clave}

Formación de maestros, escuelas normales, cambio educativo.

\section{Introducción}

La presente investigación parte de la preocupación de maestros formadores de docentes que desde una perspectiva amplia e incluyente se han planteado conocer el posicionamiento que tienen los maestros en formación de tres escuelas normales del estado de Chihuahua en relación con el cambio educativo.
Para abordar este estudio se realizaron varias acciones muy significativas y precisas, que permitieron llevar a cabo la investigación, documentar los procesos, evaluar los resultados, encontrar conclusiones válidas con respecto a la pregunta de investigación y finalmente encontrar puntos importantes para realizar algunas propuestas como parte producto del análisis de los datos recolectados 
y contrastados con la literatura que así lo fundamenta.

\section{Antecedentes}

Los sistemas educativos en todo el mundo enfrentan hoy en día una serie de demandas derivadas de las nuevas necesidades y expectativas sociales de las comunidades que los albergan. En los últimos 20 años ha habido cambios importantes en lo político, en lo económico, en lo social y cultural, como efecto de un fenómeno global que, a falta de un mejor nombre, se identifica como "postmodernismo" (Hargreaves, 1996). Estos cambios en el contexto resultan en nuevas expectativas que tiene la sociedad con respecto a sus escuelas.

A estas demandas ampliadas de la comunidad se agregan las nuevas formas de enseñanza y aprendizaje que se han ido desarrollando en los últimos 30 años, donde se destacan, por un lado, los planteamientos constructivistas relacionados con el aprendizaje y la enseñanza (ver, por ejemplo, a Eggen y Kauchak, 2001, y; Díaz-Barriga Arce y Hernández Rojas, 2002). Estos nuevos enfoques han cambiado sustancialmente nuestra forma de entender y ejercer la docencia. Un segundo cambio importante en relación al trabajo que se realiza en las escuelas es la integración (o por lo menos los intentos de integración) de las nuevas tecnologías de información y comunicación al trabajo educativo (Brünner, 2003; Burbules y Callister, 2001; Tedesco, 2000).

Estos cambios en el contexto educativo han sometido a los sistemas educativos, públicos y privados, a múltiples presiones para modificar lo que ofrecen a las comunidades, y la forma en la que estos ofrecimientos se hacen. Aunque cambios en el contexto siempre han resultado en exigencias de cambio hacia el interior de las escuelas, podemos decir que en los últimos 20 años estas exigencias se han multiplicado exponencialmente. Si antes se consideraba deseable que las escuelas cambiaran, ahora es una exigencia permanente que se genera en los ámbitos más diversos. Independientemente del mérito que tengan estas exigencias de cambio, sea en términos técnicos, prácticos o éticos, o de su viabilidad a corto, mediano o largo plazo, es un hecho que las escuelas, actualmente, ven el cambio educativo como un evento permanente que afecta su funcionamiento diario (Hargreaves, 1996).

Sin embargo, el cambio educativo no es, por mucho, una tarea sencilla que lleva siempre a mejores resultados, a pesar de la retórica que rodea los múltiples intentos de innovación que se llevan a cabo en diferentes países. Fullan (2002), quien es probablemente la mayor autoridad en cambio educativo en estos momentos, nos indica que de todas las iniciativas de cambio planeado que se tratan de llevar a cabo en educación, el 85\% fracasan total o parcialmente. Este hecho no es privativo de la educación. En general, el cambio organizacional no lleva a los resultados esperados en el sector privado (Burke, 2007) o en el público (Arellano, Cabrero y del Castillo, 2003).

Esta tesis presenta los resultados de una de cuatro investigaciones realizadas como parte de un proyecto de investigación cuyo propósito es responder a la siguiente pregunta:

¿Qué significado le dan los maestros en formación al cambio educativo?

Se parte del supuesto que la formación del maestro puede, en cierta forma, influir sobre la forma en la que los maestros, de acuerdo a Fullan (2002) y Hargreaves (1996) le dan significado al cambio educativo. Aunque es cierto que los maestros en formación no pueden ser considerados completamente como maestros, también es cierto que en los 4 años o más años que dedican a su formación 
se pretende que se vayan socializando a la profesión docente. No pueden considerarse simplemente como "no-maestros". Aunque al principio del proceso de formación podemos suponer que tendrán un mínimo de características similares a los maestros en ejercicio, a medida que van transcurriendo los años que dura el proceso de formación es factible suponer que irán asemejándose más, en la forma en la que perciben y actúan sobre la realidad, con aquellos que ya ejercen la profesión. Siendo así, es posible que algunos rasgos que tienen los maestros en ejercicio en relación al significado que le dan al cambio educativo empiecen a presentarse durante el proceso de formación.

Para efectos del proyecto de investigación del que se deriva la tesis que se presenta, se considera como "maestro en formación" a aquella persona matriculada a un programa de formación inicial de maestros (en el caso de México, por ejemplo, un alumno o alumna de alguna Escuela Normal Básica) que en el momento del estudio haya completado por lo menos el $50 \%$ de los créditos o cursos requeridos. Se parte del supuesto que estos "maestros en formación" ya se han socializado en cierta medida con la profesión del maestro, a diferencia del alumno que acaba de empezar el programa. Y que entre más cerca estén de completar el programa, mayor socialización habrá. Normalmente en programas de formación docente alumnos que han completado 50\% del programa o más, se encuentran, de diferentes maneras, a realizando prácticas en escuelas como maestros.

\section{Marco Teórico}

El contenido de este capítulo versa sobre la literatura que esencialmente es la base de la fundamentación teórica del problema sobre el cual se está trabajando, en ella se encuentran algunas aseveraciones interesantes que dan explicación al tema que se está desarrollando básicamente se encuentra desplegada de acuerdo al modelo de investigación expuesto en el capítulo anterior.

El cambio educativo, ¿Está en la formación de los profesores?

Tradicionalmente el sistema educativo mexicano en todos sus niveles genera un modelo educativo en el que los estudiantes en formación están regidos bajo un programa de estudios en el cual están claramente establecidos los propósitos, enfoques, las formas de evaluación, metodologías así como las actividades que llevarán a la consecución de los mismos.

La formación de los nuevos profesores no escapa de este modelo preestablecido y entonces pues ellos también deben acatar este planteamiento para que al término de su formación hayan alcanzado los rasgos deseables del perfil de egreso que el programa marca en su fundamentación teórico práctico.

El modelo educativo viene implementado en una especie espiral en el que se combinan la teoría, la reflexión, la práctica, la reflexión al irse sucediendo estos acontecimientos se van generando a la vez los retos que se deben ir superando conforme se avanza en el conocimiento el alumno puede o no tener conocer personalmente a sus asesores.

Finalmente el modelo escolarizado en el que los alumnos se encuentran desarrollando de manera puntual y a través del ciclo escolar las materias o asignaturas que establece su programa de estudios, sus maestros tienen la cronometración de actividades, periodos de evaluación y prácticas de acuerdo a lo que establece por un lado la propia curricula y por otro el sistema organizacional de la institución.

Díaz Barriga y Lugo (2003), explican sobre los términos modelo, propuesta y tendencia curricular lo siguiente: Modelo curricular, es una construcción teórica de algún objeto, describe su funcionamiento y le permita explicarlo e intervenir en él. 
RECIE. Revista Electrónica Científica de Investigación Educativa Vol. 1, núm. 1, enero-diciembre 2012, pp. 177-183.

Una tendencia se describe como la dirección de un movimiento que cobra fuerza $y$ predomina en un momento determinado. Casarini (2001) señala tres categorías de análisis del currículo. El currículo formal que se forma con el plan de estudios y los programas de cada asignatura, dice que son documentos guías que prescriben las finalidades, contenido y acciones que el maestro y sus alumnos deben llevar a cabo.

Estos modelos a su vez están afectados por el enfoque formativo en el que están sustentados, esto es, sobre la línea del pensamiento o teoría lo cual a su vez determina el tipo de práctica, el rol del maestro, el rol del alumno, los rasgos del perfil de egreso, que permiten definir el tipo de maestro que se está formando.

\section{Enfoque metodológico}

Para la realización de este documento se realizó investigación con estudio de casos de Stake (1999). La cual estudia una situación detalladamente proporcionando una teoría naturalista de la situación. Dado que los maestros en formación participantes en la implementación de esta innovación nos permitieron dar un seguimiento y aun análisis a sus descubrimientos, interacciones y constructos con relación al cambio educativo.

Según Erickson, la característica más distintiva de la indagación cualitativa es el énfasis en la interpretación, dando gran importancia a la participación del investigador y a la recogida de los datos de manera objetiva, y que a la vez reoriente la observación para precisar o sustanciar esos significados y en donde, además, las preguntas de investigación se pueden ir modificando conforme avanza la investigación, en un enfoque progresivo (Stake, 1999). En la investigación cualitativa las explicaciones están un tanto alejadas de las explicaciones de las causas y efectos de los fenómenos, aunque estos guardan una estrecha relación entre sí, no obstante las generalizaciones no tienen que ver con estos principios.

\section{Contextualización}

Una de las instituciones es la Escuela Normal rural Ricardo Flores Magón, ubicada en Ciudad Saucillo, dicha institución se encuentra a orillas de la Ciudad, en una zona rural, es un internado para señoritas, los servicios con que cuenta son de tipo asistencial, el nivel socioeconómico de las estudiantes es bajo, por lo que reciben una beca por parte de gobierno, que les permite que todo lo que se les otorga en la institución sea de manera gratuita.

La Normal "Ricardo Flores Magón" de Saucillo ofrece la licenciatura en educación Preescolar y primaria, en ambas Licenciaturas sólo se atiende a mujeres, cuenta con una población estudiantil de 510 alumnas, repartidas en 12 grupos de primero a quinto semestre y once equipos de séptimo semestre.

Otra de las escuelas participantes es la Benemérita y Centenaria Escuela Normal del Estado profesor Luis Urías Belderraín, ubicada en la capital del Estado, atiende alumnos de ambos sexos, en su totalidad son 856, distribuidos en 24 grupos de primero a sexto semestre y 24 equipos de octavo semestre;

La tercera es Normal Miguel Hidalgo, ubicada en Ciudad Parral, ubicada en una zona urbana, atiende las Licenciaturas en educación primaria y Preescolar y en este ciclo escolar incursiona con una nueva Licenciatura especializada para la población indígena, con un total de 420 alumnos, Las técnicas de investigación.

La Observación. En las ciencias humanas, como lo menciona Giroux y Tremblay (2004) la técnica de la observación es asimismo muy útil. De acuerdo a Quivy y Campenhoudt (en Giroux y Tremblay, 2004) esta técnica se ajusta perfectamente al análisis de lo no verbal y de lo que éste revela: las conductas instituidas y los códigos de 
comportamiento, la relación con el cuerpo, los modos de vida y los rasgos culturales, la organización espacial de los grupos y de la sociedad, etcétera. Según Giroux y Tremblay existen dos grandes formas de observación, la primera es la observación en un medio controlado, a la que se recurre sobre todo en psicología. Guía de observación sistemática no participante: porque en el presente caso la investigadora no pertenece a ninguno de los grupos en estudio, y permite registrar los acontecimientos que suceden en el interior de las escuelas y sus dinámicas en donde tanto los docentes como los alumnos, actúan de forma normal y cotidiana. Para este estudio se utilizará un registro de observación sistemática y no participante, misma que muestra el grupo del docente observado,

Una de las técnicas que caracteriza el trabajo de interaccionismo en educación ha sido definida, como observación participativa. Se elaboraron dos versiones de registros de observación para estudiar al mismo actor para que figuren como recopilaciones variadas de representaciones y acciones de los participantes en un mismo contexto y escenario con cierto grado de validez.

\section{Resultados}

Los resultados obtenidos en los instrumentos que se aplicaron a ciento veinte alumnos en formación tomados como muestras de las tres escuelas normales públicas del estado de Chihuahua, ellos se encuentran estudiando las Licenciaturas en educación preescolar y primaria, para dar respuesta a la pregunta de investigación, ¿Qué significado le dan los maestros en formación al cambio educativo? primero se abordan los cuestionamientos que se establecieron para las entrevistas y se relacionan con los encuentros de análisis que permitieron encontrar elementos que dan sustento a dicha pregunta.

Se parte del análisis que implica la formación docente de los alumnos que forman parte de esta investigación, el cual se realiza a través de la observación, en primer término se debe mencionar que los alumnos están pre determinados a desarrollar un programa de estudios que ya fue diseñado ex profeso para la carrera que cursan, en donde los objetivos y metas que habrán de alcanzar los convierte en personas cautivas de un currículo preestablecido, que si bien es cierto los docentes tienen la libertad de cátedra, también es cierto que los rasgos deseables del perfil de egreso también ya están preestablecidos, entonces pues la institución destina el tiempo y el espacio en donde maestros y alumnos deben desarrollar sus actividades profesionales para alcanzar los propósitos consabidos por esta comunidad escolar.

Las propuesta de innovación de cualquier índole se ven socavadas por toda esa serie de condicionantes que se generan al interior de cada centro educativo, situaciones que inciden en los escenarios de la formación docente y dan figura al significado que le dan al cambio educativo los maestros en formación.

Los maestros en formación fueron cuestionados en relación con el concepto que tienen del cambio educativo, la respuesta que ellos proporcionan en este sentido evidencia que tiene un significado especial pues da pauta a la transformación, a la renovación de lo ya establecido.

"El cambio educativo es algo que se lleva a cabo con el fin de mejorar la calidad que hay en la educación, sin embargo para darse este cambio se debe tener una capacitación, se debe investigar para así innovar y lograr una mejora"

"Bueno pues al hablar de cambios estamos hablando de una reconstrucción o de agregarle o quitarle algo a lo que ya esta impuesto, creo que se le está llamando un cambo educativo un tanto por los políticos que están ahorita, no le veo en sí mucho 
cambio, yo le llamaría una reconstrucción no un cambio, porque al hablar de cambio es como que quitar lo que está y poner algo nuevo, sería reconstrucción o agregar o complementar lo que está, no en sí un cambio."

"Después de tantos exámenes y estar analizando como está la educación en México, no se ha logrado mucho después de tantos años que tenemos con ese programa, se volvió como que haber que podemos hacer para que las cosas sean diferentes, entonces yo digo que si se trabaja como lo están planteando podemos tener muy buenos resultados, claro que no se van a ver en uno o dos años, estamos hablando de una generación a lo mejor 8 ó 9 años que ya sea un proceso completo que abarque desde preescolar hasta la educación primaria, yo digo que si trae muchos retos para el docente porque es sobre quién va a recaer esta responsiva de llevarlo a cabo como se debe planear porque creo que todos los programas han sido así, se deja a consideración del docente y no es que el programa funcione o no sino que el profesor lo eche a funcionar o no en sus grupos".

Estas son palabras textuales sobre algunas de las preguntas que se hicieron a los maestros en formación.

Sobre los resultados al primer cuestionamiento realizado, las respuestas se concentraron en el hecho de ver que el cambio educativo se puede generar a través de todas y cada una de las innovaciones, transformaciones, propuestas, reformas que se implementen al interior de la escuela normal y que posibiliten la participación de todo el colectivo escolar, pero que además estas sean encaminadas a una mejora tanto en la formación como en la implementación de nuevas estrategias de trabajo que se vean reflejadas en el trabajo docente que se realiza para con los estudiantes y que se pueda evidenciar también en la práctica educativa del futuro maestro en la escuela preescolar o primaria en donde realiza su trabajo académico. "La innovación es multidimensional. En la realización de cualquier programa o políticas nuevos, entran en juego por lo menos tres componentes: uso de materiales nuevos, el posible uso de nuevos sistemas de enseñanza y las posibles alteraciones de las convicciones." (Fullan 2002: 42).

Las respuestas a esta pregunta evidencian que los maestros en formación consideran que el cambio educativo puede darse a través de la confrontación de ideas con otros maestros y alumnos y que en este intercambio se puede generar el conocimiento de diferentes necesidades pedagógicas incluyendo el uso de las tecnologías y del internet el cual puede posibilitar o dar otra perspectiva es este tipo de intercomunicación.

Los futuros maestros entrevistados consideran de gran importancia el desarrollo de sus competencias para poder generar el cambio educativo y desarrollar a su vez una educación de calidad en donde los niños y las niñas que estén a su cargo en el acto educativo, puedan desarrollarse como personas más reflexivas y críticas.

Los recursos tecnológicos que están al alcance de los maestros en formación proporcionan herramientas que pueden ser utilizadas para que tanto alumnos como maestros puedan mejorar sus prácticas educativas, esto posibilita que tiempo muy corto puedan estar en contacto con materiales, recursos bibliográficos, revistas, etc., así como con otros maestros en formación y formadores de docentes generando oportunidades de cambio e intercambio.

El cambio educativo para los maestros en formación desde su punto de vista genera 
tanto ventajas como desventajas, se abordarán las primeras, considerando que éstas permiten visualizar el significado que para ellos representa el cambio educativo.

\section{Referencias}

Casarini Ratto, Martha, Teoría y diseño curricular, (2004) Ed. México, Trillas, Instituto Tecnológico y de Estudios Superiores de Monterrey, Universidad Virtual.

Díaz-Barriga Arceo, F., \& Hernández Rojas, G. (2002). Estrategias docentes para un aprendizaje significativo. Una interpretación constructivista (2a. ed.). México, D.F.: McGraw-Hill.

Flores Kastanis, E., Flores Fahara, M., García Quintanilla, M., Rodríguez Bulnes, G., Holguín Ruiz, L. T., Olivas Maldonado, M., et al. (2007). Las escuelas públicas como Comunidades de Aprendizaje (Reporte de Investigación No. 3). México, D.F.: Dirección General de Desarrollo de la Gestión e Innovación Educativa de la Subsecretaría de Educación Básica.
Fullan, M. (2002). Los nuevos significados del cambio en la educación (A. Solà \& C. Sánchez, Trads. Vol. 14). Barcelona: Octaedro.

Fullan G Michael y Hargreaves, Andy, La escuela que queremos (Los objetivos por los que vale la pena luchar), primera edición en la biblioteca para la actualización del maestro de la SEP, 1999.

Hargreaves, A. (1996). Profesorado, cultura y postmodernidad (Cambian los tiempos, cambia el profesorado) (P. Manzano, Trad.). Madrid: Morata.

Hargreaves, A.,Lieberman, A., Fullan, M., \& Hopkins, D. (Eds.). International Handbook.

\section{Agradecimientos}

Al Dr. Eduardo Flores Kastanis por su asesoría.

Al ITESM y a la UTEP Por permitirmos estar en sus aulas y por su certificación. 
RECIE. Revista Electrónica Científica de Investigación Educativa

Vol. 1, núm. 1, enero-diciembre 2012, pp. 177-183.

Morales Sifuentes, L.A.; Gómez Heredia, B; y Pacheco Ríos, C.M. 